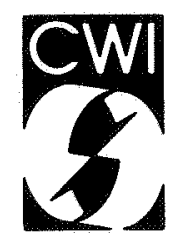

Centrum voor Wiskunde en Informatica Centre for Mathematics and Computer Science

\author{
P.M.B. Vitányi
}

Development, growth and time 
The Centre for Mathematics and Computer Science is a research institute of the Stichting Màthematisch Centrum, which was founded on February 11, 1946, as a nonprofit institution aiming at the promotion of mathematics, computer science, and their applications. It is sponsored by the Dutch Government through the Netherlands Organization for the Advancement of Pure Research (Z.W.O.). 


\title{
Development, Growth and Time
}

\author{
Paul M.B. Vitányi \\ Centre for Mathematics \& Computer Science (CWI) \\ Kruislaan 413, 1098 SJ Amsterdam, The Netherlands
}

\begin{abstract}
We propose a simple mathematical model for filamentous growth and development. The new model relates stereotype elemental (cellular) behavior to empirically observed overall growth curves. As examples we obtain the sigmoidal growth curves. Basic is the separation of subjective or physiological time of the organism from objective or absolute time and the relation between them. The underlying philosophy is related to Lindenmayer's developmental model.
\end{abstract}

1980 Mathematics Subject Classification: 92A05, 92A10, 68D20, 68J10

CR Categories: J.3, F.1.1, F.4.2 Gg L $30,6 g F 11,69 \neq 42$

Keywords \& Phrases: biological development, growth, sigmoïdal curves, elemental (cellular) behavior, physiological time, Lindenmayer sytems

Note: This paper is dedicated to the 60th birthday of professor Aristid Lindenmayer. It will appear in a special volume to be published by Springer Verlag.

\section{INTRODUGTION}

So right away I found out something about biology: it was very easy to find a question that was very interesting, and that nobody knew the answer to. In physics you had to go a little deeper before you could find an interesting question that people didn't know.'

R.P. Feynman, "Surely You're Joking, Mr. Feynman," W.W. Norton \& Co., New York, 1985.

The study of growth and form in biology has from the outset been of a mathematical nature. Indeed, it is hard to point out a subject which has been so advantageous to both sciences $[14,12,13]$. Here we want to explore a mathematical model for developmental growth in filamentous organisms. As examples we derive sigmoidal growth curves. For that purpose, the model is couched in terms of absolute time versus the time as experienced by the machinery of the organism. The first we term physical time and the second physiological time. The idea is that absolute time is that which is kept by a clock made of "dead"

Report CS-R8516

Centre for Mathematics and Computer Science

P.O. Box 4079, 1009 AB Amsterdam, The Netherlands 
matter, while the physiological time corresponds to the number of sequential events in the "living" organism. As a convenient and, in this context, appealing framework in which to model the organism's sequence of events, its developmental history so to speak, we use the automata theory model of development proposed by Lindenmayer [6]. Rather than aiming at a universal growth function, we propose a general model from which particular growth functions can be obtained at will by setting appropriate biologically significant parameters. The new paradigm would appear to embrace also growth of bulky organisms and aspects of development other than growth. However, the automata theory models are adequate only for filamentous organisms (like algae), and we restrict ourselves accordingly here. Let us briefly outline a few details of Lindenmayer's model which are pertinent to the present discussion.

Recall that $W^{*}$ is the set of all strings over alphabet $W$, including the empty string $\epsilon$ (i.e., the string with no letters). For example, let $W=\{a, b\}$. Then the set $W^{*}=\{\epsilon, a, b, a a, a b, b b, a a a, a a b, \cdots\}$. A homomorphism $\delta: W \rightarrow W^{*}$ is a mapping such that $\delta(\epsilon)=\epsilon$ and $\delta(x y)=\delta(x) \delta(y)$ for all $x_{2} y \in W^{*}$. A $D O L$ system (deterministic context-free Lsystem) $G=\langle W, \delta, w\rangle$ consists of an initial string $w$ over a finite nonempty alphabet $W$ and a homomorphism $\delta: W \rightarrow W^{*}$. Take for example $W=\{a, b\}$ as above, $\delta(a)=b$ and $\delta(b)=a b$ and the initial string $w=a$. The string sequence $S(G)$ generated by $G$ is

$$
\begin{aligned}
S(G) & =w, \delta(w), \delta^{2}(w), \delta^{3}(w), \delta^{4}(w), \cdots \\
& =a, b, a b, b a b, a b b a b, \cdots \quad \text { (for this example). }
\end{aligned}
$$

Let $|x|$ denote the length (number of letters) of a string $x$. So $|\epsilon|=0$ and $|a b b a b|=5$. The growth function $f_{G}(t)$ associated with $G$ is defined as $f_{G}(t)=\left|\delta^{t}(w)\right|$. In our example we have $f_{G}(t)=f_{G}(t-1)+f_{G}(t-2)(t \geqslant 2)$ and $f_{G}(0)=f_{G}(1)=1$. So $f_{G}(t)$ is the th Fibonacci number. The biological interpretation of all this is as follows. Suppose we start out with a filament $w$, in this case consisting of one cell of type $a$. The alphabet $W$ stands for the set of different cell types of the organism, while the homomorphism $\delta$ describes the rules for growth and development. In the example, a cell of type $a$ turns into a cell of type $b$, and a cell of type $b$ splits in a two cell filament $a b$. So $S(G)$ gives the developmental history of the filament, and $f_{G}$ its growth. The system is deterministic in the sense that a given cell type always does the same thing, and it is context-free because a cell does its thing autonomously (without interaction with adjacent cells; that is where the ' 0 ' in DOL stands for).

The growth function of a DOL system satisfies a homogeneous difference equation with constant integer coefficients. Given the homomorphism, it is easy to find the closed form solution of the growth function. Such a solution is a combination of polynomial and exponential terms of the form

$$
f_{G}(t)=\sum_{i=1}^{m} p_{i}(t) c_{i}^{t}
$$

where the $p_{i}(t)$ 's are polynomials in $t$ and the $c_{i}$ 's are algebraic constants [5]. Such functions 
cannot account for the empirically derived sigmoildal growth curves (shaped like the lower case greek letter $\sigma$ ) commonly encountered in developmental biology. Well known examples are the logistic growth function $A /\left(1+B e^{-k t}\right)$ and the monomolecular growth function $A\left(1-B e^{-k t}\right)$, see [12]. This leads to problems about the adequacy of Lindenmayer's proposal. Approximating sigmoidal functions by solutions of the form (0) is impossible except through roughshod trivialization. Viz., postulate as many different cell types, which produce each other in chains, as is necessary for the desired approximation of the initial curve, and let everything stay constant from the time that the curve does not change too much anymore. However, suppose we drop the assumption that changes (= rewriting of strings) in the system occur at unit time intervals. Then we can describe in the model phenomena like progressive dissipation of growth energy, biological rhythms and changes in environmental conditions which influence the growth rate. This is essentially a hybrid view assuming discrete cells and instantaneous cell division but continuous time. Now the number of past rewritings corresponds to physiological time and the time consumed to physical time. It will appear that exponential growth in physiological time yields logistic growth in physical time. Not only that, but linear growth in physiological time leads to monomolecular growth in physical time, thus capturing the two main sigmoidal curves by the two main types of $L$ growth. These results are but two examples of a new paradigm. Its strength is that the new model relates stereotype elemental (cellular) behavior to empirically observed overall growth curves. In an Appendix we discuss some extensions and refinements of the method.

If we want to obtain sigmoidal growth curves with the original L-systems then not even the introduction of cell interaction can help us out. In the first place, we end up with constructing quite unlikely flows of messages through the organism (see [4]) which are more suitable to electronic computers, and in fact give the organism universal computing power. Secondly, and this is more fundamental, we can not obtain growth which, always increasing the size of the organism, tends towards stability in the limit. The slowest increasing growth we can obtain by allowing cell interaction is logarithmic and thus can not at all account for the asymptotic behavior of sigmoidal growth functions like the logistic and monomolecular ones. A third objection may be that L-systems with cell interaction are highly vulnerable to disturbances: a small disturbance usually causes a completely different behavior, contrary to biological organisms which are robust enough not to be swayed from their chosen path by minor disturbances.

Truly exponential growth as in the theory of L-systems, does not reflect biological reality: in a short time the organism would fill the universe! There is not enough food stuff for that. However, it has been shown that under continuous culture conditions bacteria and monocellular algae can be kept under exponential growth as can be filamentous algae [8]. If the culture medium is not interfered with, as must eventually be the case, then there will be a sigmoidal growth curve. Growth curves of higher plants show mostly this form. Sometimes there is also a very long, nearly linear, median phase. Lianes 'grow that way. In any case, that real growth normaly stops somehow is not necessarily related to food constraints but can also be the results of higher hierarchical processes such as 
flowering.

Growth functions as occurring in developmental biology have a purely empirical origin. The size of an organism is plotted graphically against its age. The resulting curve is expressed, as accurately as need be, by means of an algebraic equation. In general, no biological significance is attributed to the exact form this equation takes. The growth function's chief function is to facilitate the analysis of the curve of growth [12]. We do assign a biological significance to the equation of the growth function. We do so by taking Lindenmayer's discrete model as a structural representation of the elemental cellular behavior and composing it with concepts from a more classical analysis of biological phenomena.

In biology, as opposed to the usual automata theoretical approaches, we meet the problem of environment. In an organism each cell has an environment (apart from the adjacent cells) which is going to influence its behavior, c.q. division rate. In algae this is the surrounding water from which it draws its food. In larger plants the environment consists of the outside world, and inside the organism, e.g., the vessels which transport nutrients. Furthermore, growth inhibitors, temperature and, for all we know, the phases of the moon will influence the growth rate of the organism. Every one of these exogenous influences may cause changes in endogenous parameters. In the biologists view [12] empirical generalizations can be made like:

(i) Size is a monotonic increasing function of age.

(ii) Usually, what results from growth is itself capable of growing.

(iii) Under the actual conditions of development living tissue progressively loses power to reproduce itself at the rate it was formed.

Automata theory does not heed these precepts but deals with abstractions which are not subject to physical constraints; there identical cells do identical things at all times. In actual organisms, differences in environment in space and time are going to create differences in cell behavior such as division rates etc. So even if we do assume that a cell is essentially an autonomous unit, in real life changes and divisions do not occur at unit time intervals, but division times are governed by environmental parameters, like concentration and accessibility of nutrients, growth inhibitors, enzymes, temperature, light. Now the usual way to obtain somewhat complicated behavior in automata models is by postulating many cell types. This seems not to be based on experimental evidence. The biologist observes very little real differences in cell types/states (e.g., cells with distinct stereotype behavior). For instance, in experiments with growth in roots of corn cobs $[1,2]$, the experimentalist can distinguish essentially between cells in the core and those in the surrounding tissue only, and insists that all cells in one of these areas behave more or less alike [3]. In some experiments on algae, a distinction is made between four cell types, and then not by observed characteristics but according to ancestry and position only [9]. If the environmental conditions are changed, these experimentalists observe no other cell types but changes in size and division times [10]. Yet, in order to account for differences in cell behavior induced by time or extracellular agents, the automata theorist is inclined to postulate a very large 
number of cell states. In doing so, he makes no distinction between the autonomous properties of cells, and changes in division times due to extracellular agents. This is were he looses the distinction between essentially different aspects, and the model becomes at best chaotic and at worst plain wrong. Opting for the best parts of two different worlds, below we assume but a few different cell types and take the intervals in between changes in the model as a variable quantity. We call the elapsed actual time physical or real time and the number of times the model has undergone changes physiological time. This seems agreeable with biological terminology. To quote [12]:
..."Growth is more rapid earlier in life than later, and if the time intervals are equal in length - are days for example - the approximation will correspondingly be less efficient at the beginning than at the end. The length of the chosen interval should evi- dently bear some relation to the work done by the organism in its life span; to the organism's "physiological age" in fact ... (Physiological time is biology's claim to be con- sidered at least as obscure to the lay mind as theoretical physics. The organism it is argued, dispenses a Time of its own making by a just measure of the work done ...)"

To be more precise about the ideas we have in mind, recall the theory of DOL growth functions, e.g. [5], at which we briefly glanced above. Imagine that the clock, which governs the discrete time rewriting of the string of cells does not tick at unit time intervals, but rather at variable time intervals corresponding with the relative slowing down or speeding up of the growth of the organism, under the influence of changes in the environmental and internal parameters, and maybe related to the number of previous rewritings. Each such variable length time interval then corresponds to the time elapsed between two consecutive rewritings of the string. That is, the time interval between the occurrences of the $t$ th and $(\iota+1)$-th elements of $S(G)$ is given by $\tau_{\imath+1}-\tau_{\imath}$ where $\tau_{\iota}$ is the time elapsed up to the occurrence of the $\iota$-th element of $S(G)$. To be able to use analytical methods we give the relation between $\iota$ and $\tau_{\iota}$ by a continuous function $t: \mathbb{R}+\rightarrow \mathbb{R}+$ (where $\mathbb{R}+$ denotes as usual the positive real numbers) such that $t(\iota)=\tau_{\iota}$ for all $\iota \in \mathbb{N}$ ( $\mathbb{N}$ denotes the set of natural numbers). By its genesis $t$ is strictly increasing on $\mathbb{N}$ and we consider only functions $t$ that are strictly increasing on $\mathbb{R}+$ as well. The function $t$ can be interpreted as mapping the physiological time $\iota$ to the physical or real time $\tau_{\imath}$. Then the size (c.q. weight or number of cells) of the modeled organism at real time $t(\iota)$ is given by $L_{G}(t(\iota))=f_{G}(\iota)$. (If complex constants enter in $f_{G}$ this can have as its effect that values of $L_{G}$ are complex for $\iota \in \mathbb{R}_{+}-\mathbb{N}$. We circumvent this difficulty by either taking $L_{G}(t(\iota))$ equal to the absolute value of $f_{G}(\iota)$ in such cases or by only ascribing a physical interpretation to $L_{G}$ and $f_{G}$ for $\iota \in \mathbb{N}$.) Since $t: \mathbb{R}+\rightarrow \mathbb{R}+$ is strictly increasing, there does also exist the inverse mapping $t^{-1}=i: \mathbb{R}+\rightarrow \mathbb{R}+$ defined by $i(\tau)=\iota$ if $t(\iota)=\tau$. Then $\lfloor i(\tau)\rfloor$ gives the number of rewritings, which took place up to time $\tau$, as a function of the elapsed real time $\tau$. (Start with the initial string at time zero.) It seems reasonable to assume that the time delay between two consecutive stages (rewritings) of an organism is related to, e.g., the concentration of nutrients it has access to and the waste products and growth inhibitors it secretes. Such concentrations will be related to the organism's size and history in that environment. So, 


$$
L_{G}(\tau)=f_{G}(i(\tau))
$$

where $i: \mathbb{R}+\rightarrow \mathbb{R}+$ is the physiological time as a function of the physical time. The function $i$ is found by describing (if advantageous by differential equations) the relations between $t(\iota), L_{G}(\tau)$, the influences of the environmental parameters which are not influenced by the organism such as temperature, day and night cycles, and the influences of the environmental parameters which are influenced by the organism such as food concentration. (To take a simple example where we do not ascribe a physical meaning to $t(\iota)$. Suppose that $f_{G}(\iota)=2^{\iota}$ and $t(\iota)=\iota^{2}$. Then $i(\tau)=\sqrt{ } \tau$ and $L_{G}(\tau)=2^{\sqrt{ } \tau}$.) One assumption we have made is that the relative changes of time intervals in between the rewriting of a letter does not depend on the letter itself or its position in the string. The theory could be extended to take care of this too, cf. Appendix.

In the sequel we denote the values $\iota$ of the function $i(\tau)$ and the function itself both by $i$ and, similarly, $\tau$ and $t$ both by $t$. Which one is meant, the function or its value, will be clear from the context. So we write $\frac{\mathrm{d} t(i)}{\mathrm{d} i}=1 / \frac{\mathrm{d} i(t)}{\mathrm{d} t}$ for $\frac{\mathrm{d} t(\iota)}{\mathrm{d} \iota}=1 / \frac{\mathrm{d} i(\tau)}{\mathrm{d} \tau}$.

\section{Sigmoïdal Growth Fungtions}

'Don't talk nonsense', said Alice more boldly: 'you know you're growing too.' 'Yes, but I grow at a reasonable pace', said the Dormouse: 'not in that ridiculous fashion.'

L. Carroll, Alice's Adventures in Wonderland, Macmillan, London, 1865.

Let us consider developmental growth in the new model. We illustrate the method on two examples. In these examples the underlying cell division patterns are supplied by the simplest of models and the postulated physical environment is highly stylized as well. We investigate algae-like organisms which \$2.1 reside in a closed environment containing an initial amount of food stuff, $\$ 2.2$ are subject to a periodic speeding up and slowing down of division rates (i.e., some sort of biological rhythm), and $\$ 2.3$, a combination of $\$ 2.1$ and $\$ 2.2$.

\subsection{Organisms in a Closed Environment with an Initial Amount of Nutrients}

A filamentous organism resides in a trough filled with water from which it draws its food, like in Figure 1. Assume that:

(i) the organism uses no food to maintain itself but only to grow;

(ii)it excretes no waste products etc. which inhibit its growth;

(iii)at all times the concentration of food throughout the trough is uniform; 
(iv)no parameters influence the growth except the concentration of food.

Let $a(t)$ be the concentration of nutrients at time $t$. As long as $a(t) \geqslant a_{0}$ the environment is optimal and the organism grows according to a DOL system, that is, physiological time and real time are the same. After some time, say $t_{0}$ time units, the food level falls to $a_{0}$ and the growth rate starts slowing down. The surface of the filamentous organism is proportional to its length (the number of cells it is made up of), i.e., the value of $L_{G}$. Therefore, we choose our differential equations as follows.

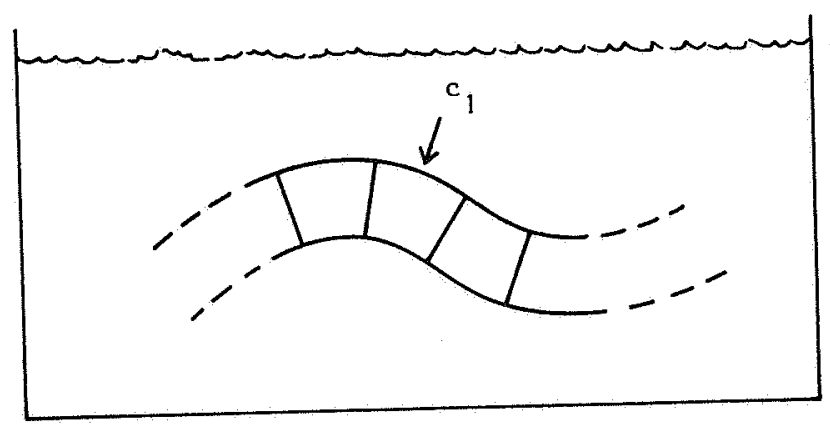

Figure 1.

For $t \leqslant t_{0}$ :

$$
\frac{\mathrm{d} a(t)}{\mathrm{d} t}=-c_{1} f_{G}(t) a(t)
$$

where $c_{1}$ is the nutrient absorption constant per unit of organism. This yields

$$
a(t)=a(0) e^{-\int_{x=0}^{t} c_{1} f_{G}(x) \mathrm{d} x},
$$

and substituting $a\left(t_{0}\right)=a_{0}$ yields $t_{0}$. From $t_{0}$ onwards the division times of cells grow larger because there is a food shortage. For $t \geqslant t_{0}$ :

$$
\begin{aligned}
\frac{\mathrm{d} a(t)}{\mathrm{d} t} & =-c_{1} L_{G}(t) a(t), \\
\frac{\mathrm{d} t(i)}{\mathrm{d} i} & =g(a(t)), \\
L_{G}(t) & =f_{G}(i(t)),
\end{aligned}
$$

for some function $g$ yet to be chosen. Since $t$ is the inverse function of $i$, equation (5) leads to

$$
\frac{\mathrm{d} i(t)}{\mathrm{d} t}=\frac{1}{g(a(t))}
$$


Considering everything in phase-space, equations (4), (6) and (7) gives

$$
\frac{\mathrm{d} a}{\mathrm{~d} i}=-c_{1} f_{G}(i) \operatorname{ag}(a)
$$

and hence

$$
\int_{\alpha=a_{0}}^{a(i)} \frac{\mathrm{d} \alpha}{\alpha g(\alpha)}=-c_{1} \int_{\imath=t_{0}}^{i} f_{G}(\iota) \mathrm{d} \iota .
$$

It is not necessary to give $a(t)$ a strong and explicit interpretation as food concentration. The fact that real growth normally stops somehow is not necessarily connected with exhaustive constraints but can also be the result of higher integrated processes such as flowering. See [7] for a discussion about largely independent levels of organization in a plant's hierarchical make up. It may be better to give $a(t)$ a more mathematical purpose than a too restricted biological significance. Assume, for instance, that integration constants may enter into $a(t)$.

\subsubsection{The Logistic Curve.}

Assume that the underlying division pattern of the organism is the D0L system $G=<\{a\},\{\delta(a)=a a\}, a>$, so $f_{G}(i)=2^{i}$. Let $g(a)=c_{2} / a$. Let $t \leqslant t_{0}$. According to (3):

$$
a_{0}=a(0) e^{-\int_{x=0}^{t_{0} c_{1} 2^{x} \mathrm{~d} x}}
$$

which yields

$$
t_{0}=\log _{2}\left[1+\frac{\ln 2}{c_{1}} \ln \frac{a(0)}{a_{0}}\right] .
$$

Let $t \geqslant t_{0}$. Substituting $f_{G}$ and $g$ in (9) yields

$$
\frac{1}{c_{2}}\left(a(i)-a_{0}\right)=-\frac{c_{1}}{\ln 2}\left(2^{i}-2^{t_{0}}\right) .
$$

Substitute $a(i)=c_{2} \frac{\mathrm{d} i}{\mathrm{~d} t}($ by $(7)$ and the definition of $g$ ) and solve $i$ in

$$
\frac{\mathrm{d} i}{\mathrm{~d} t}=\frac{a_{0}}{c_{2}}-\frac{c_{1}}{\ln 2}\left(2^{i}-2^{t_{0}}\right)
$$

via separation of $i$ and $t$,

$$
\int_{i=t_{0}}^{i} \frac{1}{A+B 2^{i}} \mathrm{~d} i=\int_{x=t_{0}}^{t} \mathrm{~d} x,
$$

with

$$
A=\frac{a_{0} \ln 2+c_{1} c_{2} 2^{t_{0}}}{c_{1} \ln 2} \quad, \quad B=-\frac{c_{1}}{\ln 2}
$$


which yields, after substitution of $y=2^{i}$,

$$
\int_{y=2^{t_{0}}}^{y} \frac{1}{A y \ln 2} \mathrm{~d} y-\int_{y=2^{t_{0}}}^{y} \frac{B}{A(A+B y) \ln 2} \mathrm{~d} y=\int_{x=t_{0}}^{t} \mathrm{~d} x .
$$

Solving $i$ in (12) we obtain

$$
i(t)=\frac{1}{\ln 2} \ln \frac{C A}{1-C B} \text { with } C=\frac{2^{t_{0}} e^{A\left(t-t_{0}\right) \ln 2}}{A+B 2^{t_{0}}} .
$$

Substituting $i(t)$ in $f_{G}(i)=2^{i}$ :

$$
\begin{aligned}
L_{G}(t) & =2^{i(t)} \\
& =\frac{-A / B}{1-1 / B C} \\
& =\frac{\frac{a_{2} \ln 2}{c_{1} c_{2}}+2^{t_{0}}}{1+\frac{a_{0} \ln 2}{c_{1} c_{2} 2^{t_{0}}} e^{-\left(\frac{a_{0} \ln 2}{c_{2}}+2^{t_{0}} c_{1}\right)\left(t-t_{0}\right)}},
\end{aligned}
$$

which is of the form $\frac{X}{1+Y e^{-k t}}:$ the logistic or autocatalytic curve.

Testing some significant values of the solution:

- For $t=t_{0}$ we obtain:

$$
L_{G}\left(t_{0}\right)=2^{t_{0}}=1+\frac{\ln 2}{c_{1}} \ln \left[\frac{a(0)}{a_{0}}\right] .
$$

- For $t \rightarrow \infty$ we obtain:

$$
\begin{aligned}
L_{G} \max & =2^{i_{0}}+\frac{a_{0} \ln 2}{c_{1} c_{2}} \\
& =1+\frac{\ln 2}{c_{1}} \ln \left[\frac{a(0)}{a_{0}}\right]+\frac{a_{0} \ln 2}{c_{1} c_{2}} .
\end{aligned}
$$

This yields the growth curve depicted in Figure 2. There, for $t \leqslant t_{0}: L_{G}(t)=f_{1}(t)=2^{t}$; and for $t \geqslant t_{0}: L_{G}(t)=f_{2}(t)=$ the above logistic growth function. The only parameters involved are $c_{1}, c_{2}, a(0)$ and $a_{0}$. 


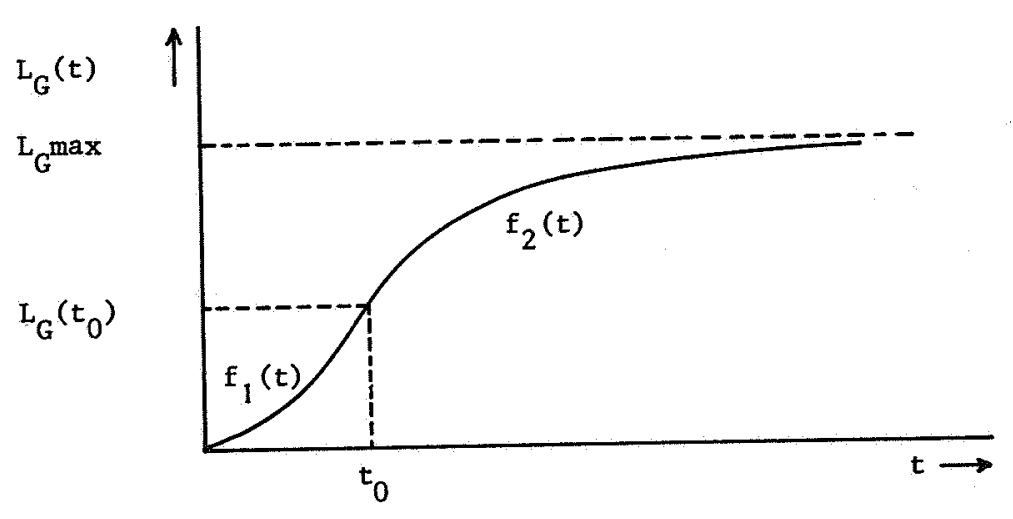

Figure 2.

\subsubsection{The Monomolecular Curve}

Assume that the underlying division pattern is $G=<\{a, b\},\{\delta(a)=a b, \delta(b)=b\}, a>$, so $f_{G}(i)=i+1$. Let $g(a)=c_{2} / a$. Then, according to (3) we can solve $t_{0}$ from

$$
a_{0}=a(0) e^{-\int_{x=0}^{t_{0}} c_{1}(x+1) \mathrm{d} x}
$$

which yields $t_{0}=-1 \pm \sqrt{1+\frac{2}{c_{1}} \ln \frac{a(0)}{a_{0}}}$. Since $\frac{a(0)}{a_{0}}$ is greater than 1 for $t_{0}>0$,

$$
t_{0}=-1+\sqrt{1+\frac{2}{c_{1}} \ln \frac{a(0)}{a_{0}}}
$$

and

$$
L_{G}\left(t_{0}\right)=f_{G}\left(t_{0}\right)=\sqrt{1+\frac{2}{c_{1}} \ln \frac{a(0)}{a_{0}}} .
$$

From (9) we see that, for $t \geqslant t_{0}$,

$$
\begin{aligned}
a(i)-a_{0} & =\frac{c_{1} c_{2}}{2}\left(\left(t_{0}+1\right)^{2}-(i+1)^{2}\right) \\
& =\frac{c_{1} c_{2}}{2}\left(L_{G}\left(t_{0}\right)^{2}-(i+1)^{2}\right) .
\end{aligned}
$$

Substituting $a(i)=c_{2} \frac{\mathrm{d} i}{\mathrm{~d} t}$ we get

$$
\frac{\mathrm{d} i}{\mathrm{~d} t}=\frac{a_{0}}{c_{2}}+\frac{c_{1}}{2}\left(t_{0}+1\right)^{2}-\frac{c_{1}}{2}(i+1)^{2}
$$

and 


$$
\int_{i=t_{0}}^{i} \frac{\mathrm{d} i}{A-B(i+1)^{2}}=\int_{x=t_{0}}^{t} \mathrm{~d} x
$$

with

$$
A=\frac{a_{0}}{c_{2}}+\frac{c_{1}}{2}\left(t_{0}+1\right)^{2}, \quad B=\frac{c_{1}}{2}
$$

which yields

$$
t=t_{0}-\frac{1}{2 \sqrt{A B}} \ln \frac{\sqrt{A / B}+\left(t_{0}+1\right)}{\sqrt{A / B}-\left(t_{0}+1\right)}+\frac{1}{2 \sqrt{A B}} \ln \frac{\sqrt{A / B}+(i+1)}{\sqrt{A / B}-(i+1)} .
$$

Set

$$
t_{0}-\frac{1}{2 \sqrt{A B}} \ln \frac{\sqrt{A / B}+\left(t_{0}+1\right)}{\sqrt{A / B}-\left(t_{0}+1\right)} \text { to } Z
$$

$\frac{1}{2 \sqrt{A B}}$ to $Y$ and $\sqrt{A / B}$ to $X$ we obtain, after some computation,

$$
L_{G}(t)=f_{G}(i(t))=i(t)+1=X\left[1-\frac{2}{1+e^{-Z / Y} e^{t / Y}}\right]
$$

and

$$
L_{G} \max =\lim _{t \rightarrow \infty} L_{G}(t)=X=\sqrt{\frac{2 a_{0}}{c_{1} c_{2}}+L_{G}\left(t_{0}\right)^{2}} .
$$

The growth curve looks like Figure 3:

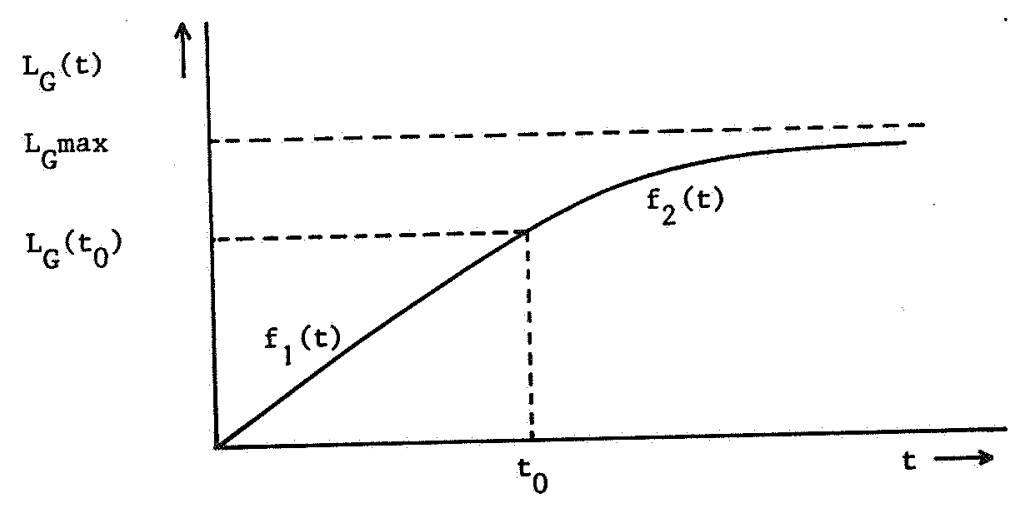

Figure 3. 
- For $t<t_{0}$ we have $L_{G}(t)=f_{1}(t)=t+1$, which is linear.

- For $t \geqslant t_{0}$ we have $L_{G}(t)=f_{2}(t)=X\left(1-2\left(1+e^{-Z / Y} e^{t / Y}\right)^{-1}\right)$.

- For $t>>t_{0}$ we obtain the the monomolecular growth curve $L_{G}(t) \approx X\left(1-2 e^{+Z / Y} e^{-t / Y}\right)$.

Significant points in the curve are:

- For $t=t_{0}$ we obtain:

$$
t_{0}=-1+\sqrt{1+\frac{2}{c_{1}} \ln \frac{a(0)}{a_{0}}}, L_{G}\left(t_{0}\right)=\sqrt{1+\frac{2}{c_{1}} \ln \frac{a(0)}{a_{0}}} .
$$

- For $t \rightarrow \infty$ we obtain:

$$
L_{G} \max =\sqrt{\frac{2 a_{0}}{c_{1} c_{2}}+L_{G}\left(t_{0}\right)^{2}}=\sqrt{\frac{2 a_{0}}{c_{1} c_{2}}+\frac{2}{c_{1}} \ln \frac{a(0)}{a_{0}}+1} .
$$

Hence we see that between the two extremes of unbounded DOL growth, viz. exponential and linear, the chosen set of differential equations which depict the depletion of food always yield a sigmoidal growth curve. Therefore, all unbounded D0L growth functions yield a sigmoidal growth curve under these conditions (for $g(a)=1 / a$ ).

\subsection{Organisms with Periodical Change of Division Rate}

In biology we meet a phenomenon called biological rhythms. Examples are circadian rhythms, florescence etc. Such phenomena might be connected with the hierarchical organization of multicellular organisms, with changes from daylight to night etc. Observations on algae show that under optimal conditions the algae behave essentially like a simple DOL system, where each transition takes place after a unit time interval of 48 hours. However, each fifth time interval the organism alternatingly skips the required transition or executes two consecutive transitions in one time interval. Thus, after each period of ten time intervals the organism reaches the stage we would expect from the DOL model, but in between it periodically speeds up and slows down its growth rate $[9,11]$. This can be stated as:

$$
L_{G}(t)=f_{G}(i(t))
$$

where $i(t)$ is the function inverse of

$$
t(i)= \begin{cases}i & \text { for } 0 \leqslant i \bmod 10<5 \\ i+1 & \text { for } 5 \leqslant i \bmod 10 \leqslant 9\end{cases}
$$

Therefore,

$$
i(t)= \begin{cases}t & \text { for } 0 \leqslant t \bmod 10<5 \\ t-1 & \text { for } 5 \leqslant t \bmod 10 \leqslant 9\end{cases}
$$


Suppose $f_{G}(i)=2^{i / 5}$. Then $L_{G}(t)=2^{i(t) / 5}$ and the growth curve is as depicted in Figure 4 .

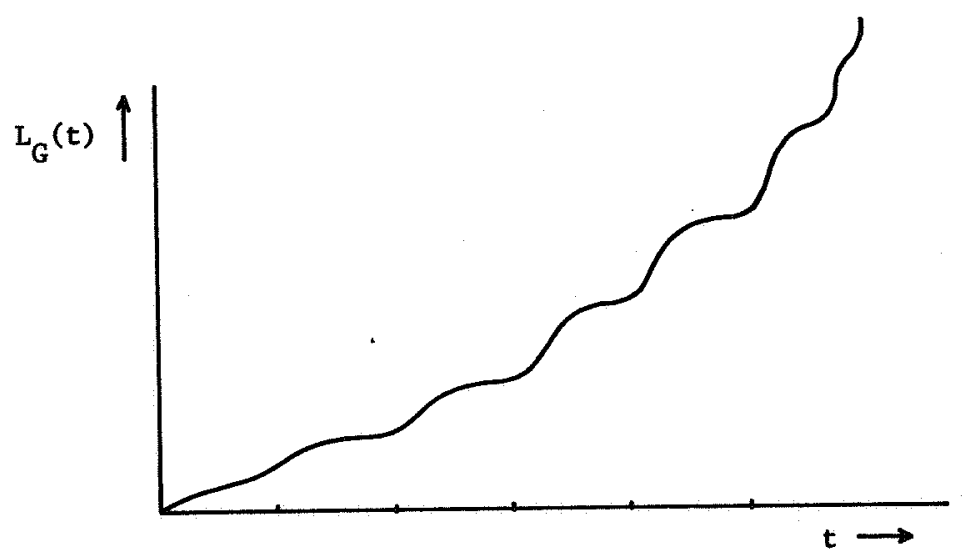

Figure 4.

\subsection{Combination of $\$ 2.1$ and $\$ 2.2$}

A combination of $\$ 2.1$ and $\$ 2.2$, i.e., an organism residing in a closed environment and showing periodic speed ups and slowing downs of growth rate, yields

$$
L_{G}(t)=f_{G}\left(i\left(i^{\prime}(t)\right)\right) \text {, }
$$

where $i$ is a function as found in $\$ 2.1$ and $i^{\prime}$ a function as found in \$2.2. The resulting growth curve looks like Figure 5, where we assume that the periodicity is independent of the organism's interaction with the environment.

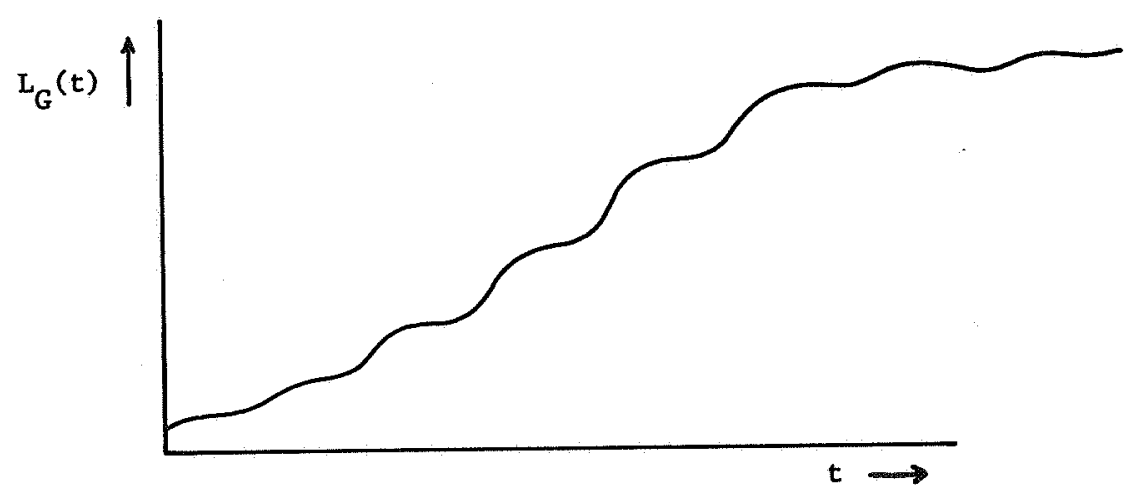

Figure 5. 


\section{REMARKS}

The universal growth equation is therefore a fiction. No amount of special pleading can override the finality of this mathematical verdict.

P.B. Medawar, in [12].

Here we have only considered filamentous organisms such as algae under the most simple cell division pattern. But it is clear that the method should be applicable to 3-dimensional growth as well. First find a, preferably context-free, model of how the organism grows in physiological time (the essential cell ancestry and division pattern) and then try to find the functional relation between physiological time and physical time. The advantages of such a procedure are that we have both one (qualitative) fundamental physiological time model and that the transition from one type of growth to another, e.g. from exponential to logistic, does not require changing the model but is a consequence of the functional relation between physiological and physical time which governs the quantitative aspects of the matter. Among experimentalists it is considered that the overall approximations like exponential, logistic etc. growth curves have nothing to do with elemental (cellular) behavior. Furthermore, usually only the initial exponential stage is studied; the latter stages of growth are more or less neglected. We have tried to establish a relation between elemental behavior and the overall growth curve and we have introduced as a most significant state of a growing organism, or of the history of a growing organism, the stage at which the growth ceases to be exponential and becomes sigmoidal: time $t_{0}$. These ideas should be useful to experimentalists trying to fit theoretical growth functions to observed data. There is no shortcut to such meaningful growth curves; the best we can hope for is to supply a convenient framework in which to express and sensibly separate the influences of the various biological aspects which have to be taken into consideration.

\section{Appendix: Extensions AND Refinements}

The assumption that the relation between physiological time and physical time is the same for all cell types in the organism can be relaxed to obtain a more general model where $a$ is rewritten as $f(t, a) \in\{a, \delta(a)\}, a \in W$ and $t \in \mathbb{N}$. We can then define the growth matrix at time $t$ as*

$$
M_{G}(t)=\left(\begin{array}{c}
\overline{f\left(t, a_{1}\right)} \\
\overline{f\left(t, a_{2}\right)} \\
\cdots \\
\overline{f\left(t, a_{n}\right)}
\end{array}\right] \text { with } W=\left\{a_{1}, a_{2}, \ldots, a_{n}\right\}
$$

* If $x$ is a string over alphabet $W=\left\{a_{1}, a_{2}, \ldots, a_{k}\right\}$ then $\bar{x}=\left(x_{1}, x_{2}, \ldots, x_{k}\right)$ where $x_{i}$ is the number of occurrences of letter $a_{i}$ in $x$. So, for alphabet $W=\{a, b\}$, we have $\bar{a}=(1,0), \overline{a b b a b}=(2,3)$ and $\bar{\epsilon}=(0,0)$.

$\dagger$ If $\bar{v}$ is a row vector (the elements written left to right) then $\bar{v}^{T}$ (v transposed) is the corresponding column vector (the elements written top to bottom). 
where $\overline{f\left(t, a_{i}\right)}$ will be either $\bar{a}_{i}$ or $\overline{\delta\left(a_{i}\right)}$, depending on $t$. In terms of the notions about growth functions as in [5], we would say that $M_{G}(t)$ is either the unit matrix $I$ or the 'classic' growth matrix $M_{G}$, depending on $t$. We then define†

$$
f_{G}(t)=\bar{w} M_{G}(1) M_{G}(2) \cdots M_{G}(t)(1,1, \ldots, 1)^{T} .
$$

This is useful to express different division times of different cell types without having to introduce different cell states to account for distinct delays in division rates. We could even go further, and use the DTOL mode. Recall that a DTOL system (deterministic context-free table L-system) is a triple $G=\left\langle W,\left\{\delta_{1}, \delta_{2}, \ldots, \delta_{k}\right\}, w>\right.$ such that for all $i, 1 \leqslant i \leqslant k$, $G_{i}=\left\langle W, \delta_{i}, w\right\rangle$ is a D0L system [4]. A control word $u$ is an element of $\{1,2, \ldots, k\}^{*}$. A word $v$ is said to derive a word $v^{\prime}$ in $G$ under the control word $u=i_{1} i_{2} \cdots i_{l}$ if

$$
v^{\prime}=\delta_{i_{i}} \delta_{i_{l-1}} \cdots \delta_{i_{2}} \delta_{i_{1}}(v)
$$

Define for a finite set $A=\left\{M_{G}(t) \mid t \in \mathbb{N}\right\}$ a DTOL system

$$
G=\left\langle W,\left\{\delta_{1}, \delta_{2}, \ldots, \delta_{k}\right\}, w\right\rangle
$$

where $k$ is the number of elements in $A$ and each table $\delta_{i}$ corresponds to the distinct element of $A$ for which it is the associated set of rewriting rules, i.e., $A=\left\{M_{G_{1}}, M_{G_{2}}, \ldots, M_{G_{k}}\right\}$, where

$$
M_{G_{i}}=\left(\begin{array}{c}
\overline{\delta_{i}\left(a_{i}\right)} \\
\overline{\delta_{i}\left(a_{2}\right)} \\
\cdots \\
\overline{\delta_{i}\left(a_{n}\right)}
\end{array}\right)
$$

for all $i, 1 \leqslant i \leqslant k$. Now we can define a computable function $h: \mathbb{N} \rightarrow\{1,2, \ldots, k\}$ with as argument the real time $t$ and which is composed from functions which compute from the relevant parameters which table $\delta_{h(t)}$ is applicable at time $t$. Then the string sequence

$$
S_{h}(G)=w, \delta_{h(1)}(w), \delta_{h(2)} \delta_{h(1)}(w), \ldots, \delta_{h(t)} \delta_{h(t-1)} \cdots \delta_{h(1)}(w), \cdots
$$

gives us the required developmental history of the modeled organism and the lengths of the successive elements of $S_{h}(G)$ give us the associated real time growth function:

$$
f_{G, h}(t)=\bar{w} M_{G}(h(1)) M_{G}(h(2)) \cdots M_{G}(h(t))(1,1, \ldots, 1)^{T} .
$$

Example. Suppose we have $G=<\{a\},\left\{\delta(a)=a^{2}\right\}, a>$ and $f(i)=2^{i}$. If $t=i^{2}$, then $L_{G}(t)=2^{\sqrt{ } t}$. The approach above models the organism as follows.

$$
G^{\prime}=<\{a\},\left\{\left\{\delta_{1}(a)=a\right\},\left\{\delta_{2}(a)=a^{2}\right\}\right\}, a>
$$

Hence $M_{G_{1}}=(1), M_{G_{2}}=(2)$ and

$$
h(t)= \begin{cases}2 \text { if } t \text { is a square } \\ 1 \text { if } t \text { is not a square }\end{cases}
$$


which yields $L_{G}(t)=2^{\lfloor\sqrt{ } t}$.

We might note here that the approach taken in the previous $\S \S$ in this paper always leads to DT0L systems with two tables: if the physiological $L$ system was $G=\langle W, \delta, w\rangle$ then the associated DTOL system will be $G^{\prime}=<W,\left\{\delta_{1}, \delta_{2}\right\}, w>$ where $\delta_{1}$ is the identity homomorphism and $\delta_{2}=\delta$. The associated function $h$ satisfies

$$
h(t)=\left\{\begin{array}{l}
2 \text { if } t=t(i) \text { for some } i \in \mathbb{N} \\
1 \text { if } t \neq t(i) \text { for all } i \in \mathbb{N}
\end{array}\right.
$$

As a further extension of the ideas presented here we could make the choice of the table, for rewriting a letter at time $t$, dependent on the geometric position in the string of that occurrence of the letter. For instance, the tip of a root grows while the basal part does not. In this case, as elsewhere in this $\S$, not only the derived string sequence could be different from that of the underlying D0L system, but also the set of derived strings could differ from that of the underlying DOL system which does not happen with the approach in $\S 2$. The model is by now quite versatile and, hopefully, applicable.

\section{REFERENCES}

[1] Erickson, R.O. and K.B. Sax, "Elemental growth rate of the primary root of Zea mays," Proc. American Philosophical Society, vol. 100, pp.487-498, 1956.

[2] Erickson, R.O. and K.B. Sax, "Rates of cell division and cell elongation in the growth of the primary root of Zea mays," Proc. American Philosophical Society, vol. 100, pp.499-514, 1956.

[3] Erickson, R.O., Private communication, 1975.

[4] Herman, G.T. and G. Rozenberg, Developmental Systems and Languages. Amsterdam:NorthHolland, 1975.

[5] Herman, G.T. and P.M.B. Vitányi, "Growth functions associated with biological development," American Mathematical Monthly, vol. 83, pp.1-15, 1976.

[6] Lindenmayer, A., "Mathematical models for cellular interactions in development, Parts I \& II," Journal of Theoretical Biology, vol. 18, pp.280-299\&300-315, 1968.

[7] Lück, H.B., "Sur la traduction morphogénétique de la cinétique cellulaire pendant la croissance des entre-noeuds de Tradeacantia Flumensis Vel.," Bull. de la Société Botanique de France, pp.220-225, 1966.

[8] Lück, H.B., Private communication, 1975.

[9] Lück, H.B. and J. Lück, "Cell number and cell size in filamentous organisms in relation to ancestrally and positionally dependant generation times," pp. 109-124 in Automata, Languages, Development, ed. A. Lindenmayer and G. Rozenberg, North-Holland, Amsterdam (1976).

[10] Lück, H.B., Private communication, 1977.

[11] Lück, H.B., Private communication, 1977.

[12] Medawar, P.B., "Size, shape and age," in Essays on Growth and Form, ed. W.E. Le Gros Clark and P.B. Medawar, Clarendon Press, Oxford (1945). 
[13] Sandland, R.L., "Mathematics and the growth of organisms - Some historical impressions," The Mathematical Scientist, vol. 8, pp.11-30, 1983.

[14] Thompson, D'A. W., On Growth and Form, 2nd Edition. Cambridge University Press, 1942. 
